\title{
Avaliação das atividades antioxidante e gastroprotetora de Moringa oleifera
}

\author{
Evaluation of antioxidant and gastroprotector activities of Moringa oleifera
}

Evaluación de actividades antioxidantes y gastroprotector de Moringa oleifera

Fernanda Silva Galdino

ORCID: https://orcid.org/0000-0002-5921-8683

Faculdade Santa Maria, Brasil

E-mail: galdinofernanda02@gmail.com

Raimunda Leite de Alencar Neta

ORCID: https://orcid.org/0000-0002-4830-9854

Faculdade Santa Maria, Brasil

E-mail: $20172002001 @$ fsmead.com.br

Higor Braga Cartaxo

ORCID: https://orcid.org/0000-0001-6722-6125

Faculdade Santa Maria, Brasil

E-mail: cartaxoh810@gmail.com

Jalles Dantas de Lucena

ORCID: https://orcid.org/0000-0002-0865-9374

Faculdade Santa Maria, Brasil

E-mail: jallesdantas@gmail.com

Danielle Rocha Silva

ORCID: https://orcid.org/0000-0002-2949-2008 Faculdade Santa Maria, Brasil E-mail: prof.daniellerocha@gmail.com

Gislayne Tacyana Santos Lucena

ORCID: https://orcid.org/0000-0003-1833-9208 Faculdade Santa Maria, Brasil

E-mail: gislayne.lucena@hotmail.com

George Souza Feitoza

ORCID: https://orcid.org/0000-0001-8534-7334 Universidade Federal de Pernambuco, Brasil

E-mail: georgesouza1@ hotmail.com

Samara Alves Brito

ORCID: https://orcid.org/0000-0003-0629-7936 Faculdade Santa Maria, Brasil

E-mail: 000604@fsmead.com.br

\section{Resumo}

Moringa oleifera é uma espécie de planta arbórea e suas diferentes partes são utilizadas na medicina popular como antiinflamatório, no tratamento de doenças respiratórias e do trato gastrointestinal. Este estudo tem como objetivo realizar uma triagem fitoquímica do extrato etanólico das folhas de Moringa oleifera e avaliar sua atividade antioxidante e gastroprotetora. A triagem fitoquímica foi realizada pela dosagem de fenol e flavonóides totais, a atividade antioxidante foi avaliada em modelos in vitro por metodologias de peroxidação 2,2-difenil-1-picrilhidrasil (DPPH) e a atividade gastroprotetora foi avaliada pela metodologia de indução aguda de úlcera gástrica usando etanol PA. O teor de fenol para o extrato do estudo foi calculado como 128,4+14,42 mg EAG/g de extrato. O conteúdo de flavonóides era mg EQ/100 g extrato. Todas as concentrações do extrato exibiram atividade antioxidante, sendo a maior capacidade de sequestro de radicais livres atribuída a uma concentração de $1000 \mathrm{mg} / \mathrm{mL}$ com 71,39 $\pm 0,52$. No estudo da atividade gastroprotetora, a dose de $200 \mathrm{mg} / \mathrm{kg}$ apresentou uma inibição da lesão ulcerativa de 48,48\% em relação ao controle. Os testes de toxicidade aguda não mostraram alterações significativas entre o grupo controle negativo e o grupo tratado com EEMo na dose de $1000 \mathrm{mg} / \mathrm{kg}$. Os resultados mostram que o extrato etanólico das folhas de Moringa oleifera possui atividade gastroprotetora, possivelmente devido ao alto teor de fenóis, flavonóides e atividade antioxidante presentes no extrato.

Palavras-chave: Moringa oleifera; Gastroproteção; Antioxidante; Úlcera péptica.

\section{Abstract}

Moringa oleifera is a tree plant species and its different parts are used in folk medicine as anti-inflammatory, in the treatment of respiratory diseases and gastrointestinal tract. This study aims to perform a phytochemical screening of the ethanolic extract of Moringa oleifera leaves and evaluate its antioxidant and gastroprotective activities. Phytochemic a screening was performed by phenol and total flavonoid dosage; antioxidant activity was evaluated in in vitro models 
by 2,2-diphenyl-1-picrylhydrazil (DPPH) peroxidation methodologies and gastroprotective activity was evaluated. by the acute induction methodology of gastric ulcer using ethanol PA The phenol content for the study extract was calculated as $128.4+14.42 \mathrm{mg}$ EAG/g extract. The flavonoid content was mg EQ / $100 \mathrm{~g}$ extract. All concentrations of the extract exhibited antioxidant activity, with the highest free radical sequestration capacity being attributed to a concentration of $1000 \mathrm{mg} / \mathrm{mL}$ with $71.39 \pm 0.52$. In the study of gastroprotective activity, the dose of $200 \mathrm{mg} / \mathrm{kg}$ presented an inhibition of ulcerative lesion of $48.48 \%$ in relation to the control. Acute toxicity tests did not show significant changes between the negative control group and the group treated with EEMo at a dose of $1000 \mathrm{mg} / \mathrm{kg}$. The results show that the ethanolic extract of Moringa oleifera leaves has gastroprotective activity, possibly due to high content of phenols, flavonoids and antioxidant activity present in the extract.

Keywords: Moringa oleifera; Gastroprotevtive; Antioxidant, Peptic ulcer.

\section{Resumen}

Moringa oleifera es una especie de planta arbórea y sus diferentes partes se utilizan en la medicina popular como antiinflamatorio, en el tratamiento de enfermedades respiratorias y del tracto gastrointestinal. Este estudio tiene como objetivo realizar un cribado fitoquímico del extracto etanólico de hojas de Moringa oleifera y evaluar sus actividades antioxidantes y gastroprotectoras. El cribado fitoquímico se realizó mediante dosis de fenol y flavonoides totales, la actividad antioxidante se evaluó en modelos in vitro mediante metodologías de peroxidación de 2,2-difenil-1picrylhidrazil (DPPH) y se evaluó la actividad gastroprotectora. evaluado por la metodología de inducción aguda de úlcera gástrica utilizando etanol PA El contenido de fenol para el extracto de estudio se calculó como 128,4 + 14,42 $\mathrm{mg}$ EAG/g extracto. El contenido de flavonoides fue $\mathrm{mg}$ EQ/100 $\mathrm{g}$ de extracto. Todas las concentraciones del extracto exhibieron actividad antioxidante, atribuyéndose la mayor capacidad de secuestro de radicales libres a una concentración de $1000 \mathrm{mg} / \mathrm{mL}$ con 71,39 $\pm 0,52$. En el estudio de la actividad gastroprotectora, la dosis de $200 \mathrm{mg} /$ $\mathrm{kg}$ presentó una inhibición de la lesión ulcerosa del 48,48\% en relación al control. Las pruebas de toxicidad aguda no mostraron cambios significativos entre el grupo de control negativo y el grupo tratado con EEMo a una dosis de 1000 $\mathrm{mg} / \mathrm{kg}$. Los resultados muestran que el extracto etanólico de hojas de Moringa oleifera tiene actividad gastroprotectora, posiblemente debido al alto contenido de fenoles, flavonoides y actividad antioxidante presentes en el extracto.

Palabras clave: Moringa oleífera; Gastroprotector; Úlcera péptica, Antioxidante.

\section{Introdução}

Moringa oleifera apresenta-se como uma espécie vegetal arbórea que pode medir de 5 a $10 \mathrm{~m}$ de altura, popularmente conhecida como baqueta, lírio-branco, moringa, drumstrick tree, sujana e sanjina. É um vegetal que pertence à família Morigaceae pertencente ao gênero Moringa junto a outras 13 espécies de dicotiledônias (Lorenzi \& Matos, 2002; Paliwal et al., 2011).

A espécie tem origem na Índia, África, Paquistão e Arábia (Somali et al., 1984; Mughal et al., 1999). Porém, apresenta-se distribuída em diversos países da América do Norte, da América central e da América do Sul (Morton, 1991). No Brasil o cultivo da Moringa teve início em 1950, sendo cultivada principalmente na região nordeste, nos estados do Piauí, Ceará e Maranhão (Lorenzi \& Matos, 2002).

A utilização da M. oleifera na medicina popular é realizada historicamente de modo empírico para tratar enfermidades como a malária, febre tifoide, doenças parasitárias, doenças gastrointestinais, artrite, doenças de pele, hipertensão, diabetes e imunodepressão (Leone et al., 2015). Além disso, por possuir importantes componentes nutricionais como proteínas, vitamina A e C, fósforo e cálcio, também são encontrados relatos sobre a utilização dessa espécie vegetal na alimentação, com o consumo do seu fruto, folhas e vagens, e na utilização do óleo das sementes como cosmético (Jahn et al., 1986; Dahot, 1998; Ferreira et al., 2008; Popoola et al., 2013).

Estudos fitoquímicos utilizando as folhas, flores, sementes e frutos da $M$. oleifera revelaram a presença de diversas classes de metabólitos secundários com importantes atividades farmacológicas como flavonoides, ácidos fenólicos e taninos que apresentam atividades antioxidantes, anti-inflamatórias, gastroprotetora e antimutagênicas. Assim como compostos alcaloides e saponinas, apresentando atividades anti-câncer e anti-inflamatórias, além de compostos glicosinolatos com atividade anti-hipertensiva (Pal, Mukherjee \& Saha, 1995; Faizi et al., 1998; Sreelatha, 2009). 
A úlcera péptica é uma doença caracterizada pelo desenvolvimento de úlceras no trato gastrointestinal. As úlceras aparecem, prevalentemente, no estômago e na porção proximal do duodeno, mas podem, em casos mais raros, apresentarem-se no esôfago, na porção distal do duodeno e no jejuno. Essa doença pode ser causada como efeito colateral da utilização antiinflamatórios não esteroidais (AINEs), por infecção bacteriana causada por Helicobacter pylori, por consumo de álcool e de nicotina (Carvalho, 2000; Kalyanakrishnan, 2007).

Estudos do potencial gastroprotetor de produtos naturais para prevenir ou tratar condições de úlcera péptica tornam-se promissoras por conta da redução de efeitos colaterais apresentados, pela diminuição do custo financeiro ao tratamento e a facilidade de acesso às espécies vegetais por parte da população (Kangwan et al., 2014; de Mendonça et al., 2020).

A atividade antioxidante, relacionada com as classes de metabólicos secundários encontradas nos extratos de estudos feitos a partir de partes da $M$. oleífera, pode ser definida como a capacidade que alguns compostos possuem de prevenir os danos oxidativos causados por radicais livres produzidos pelo metabolismo celular ou por fontes externas. Prevenindo, consequentemente, o desenvolvimento de doenças que são produto do dano oxidativo como, por exemplo, as ulceras gástricas, doenças cardiovasculares e neurodegenerativas (Matsui et al., 2011; Kasote et al., 2015; Sharifi-Rad, et al., 2018)

Considerando o potencial farmacológico presente na Moringa oleifera, o presente estudo busca realizar um estudo fitoquímico dos componentes presentes no extrato etanólico das folhas desse vegetal, assim como a avaliação de sua atividade gastroprotetora e antioxidante.

\section{Metodologia}

Este estudo trata-se de uma investigação científica baseado no conhecimento popular da Moringa oleífera.

A coleta das folhas de Moringa oleifera foi efetuada na cidade de Cajazeiras - PB. A coleta foi realizada entre as $04 \mathrm{e}$ 05 horas da manhã, na cordenadas $6^{\circ} 52^{\prime} 32.3 \mathrm{~S} 38^{\circ} 33^{\prime} 44.1 \mathrm{~W}$.

As folhas coletadas foram processadas em uma exsicata, na qual encontra-se depositada e identificada no herbário Caririense Dárdano de Andrade-Lima na cidade do Crato - CE.

A realização do extrato etanólico das folhas da Moringa oleifera utilizou-se $50 \mathrm{~g}$ das folhas e $500 \mathrm{~mL}$ de álcool etílico, para a efetuação de uma extração a frio, de acordo com a metodologia descrita por Matos (1997). Além disso, efetuouse os testes de determinação da quantidade de fenóis totais conforme a metodologia descrita por Waterman \& Mole (1994) e o método para a quantificação de flavonoides totais foi realizado de acordo com o relatado por Woisky \& Salatino (1998). O teste de avaliação da atividade antioxidante foi efetuado por meio da metodologia DPPH (peroxidação do 2,2-difenil-1picrylhydrazil) de acordo com Brand-Willians, Cuvelier \& Berset (1995).

\subsection{Estudos in vivo}

Para realizar os testes de atividade gastroprotetora das folhas de M. oleifera, foram utilizados ratos Wistar machos e fêmeas, oriundos do Biotério da Faculdade Santa Maria (FSM), no município Cajazeiras - PB, com os intervalos de 12 horas de luz/escuro monitorados, mantendo-se a temperatura ambiente entre $21^{\circ} \mathrm{C}$ a $24^{\circ} \mathrm{C}$. Os ratos utilizados possuiam de 2 a 3 meses de idade, com massa corpórea entre 200 e 230 g. O projeto de pesquisa foi submetido ao Comitê de ética em utilização animal (CEUA) da Faculdade Santa Maria e aprovado com o número 04/2019

$\mathrm{Na}$ realização do teste de toxicidade, agrupou-se os ratos em dois grupos para receber, separadamente, $\mathrm{NaCl}$ 0,9\% (10 $\mathrm{mL} / \mathrm{kg}$ ) ou EEFMo $(1000 \mathrm{mg} / \mathrm{kg})$ por via oral, utilizando o método de gavagem. Após a administração, foram avaliadas a presença ou ausência de alterações comportamentais sobre o SNC e SNA e a ocorrência de óbito. As alterações comportamentais foram observadas durante as primeiras 4 horas, e por uma vez ao dia, durante 3 dias, de acordo com as diretrizes para testes de toxicidade da Oecd (2002). 
Os animais continuaram a ser observados durante 14 dias, com o consumo de água e ração medidos diariamente e com o controle do ciclo de $12 \mathrm{~h}$ luz/escuro. Após os 14 dias, os animais foram eutanasiados para ser efetuada a retirada dos seus órgãos (fígado, baço e rins) e pesagem desses.

A realização da avaliação da atividade gastroprotetora sobre um modelo agudo de úlceras grástricas induzidas por etanol P.A os ratos foram submetidos a jejum por 18 horas, após esse período, agrupou-se os animais em cinco grupos, que foram pré-tratados, por meio de gavagem, com $\mathrm{NaCl}$ - grupo controle lesionado $(0,9 \%, 10 \mathrm{~mL} / \mathrm{kg})$, lansoprazol - grupo controle positivo (30 mg/kg) e EEFMo (50, 100 e $200 \mathrm{mg} / \mathrm{kg})$.

Uma hora após as administrações, os animais receberam $4 \mathrm{~mL} / 1000 \mathrm{~g}$ de massa corporal de etanol P.A por via oral, para a indução de úlcera gástrica, segundo o modelo de Robert et al. (1979). E, após uma hora, efetuou-se a eutanasia seguido pela retirada dos seus estômagos, sendo estes abertos e colocados em recipientes com água destilada. As lesões apresentadas nos estômagos foram fotografadas e mensuradas com auxílio do software ImageJ, com os resultados apresentados em área total de lesão ulcerativa (ALU) $\left(\mathrm{mm}^{2}\right)$ em relação à área total do estômago.

Os resultados estão expressos como média \pm erro padrão da média (EPM), sendo as diferenças entre os grupos determinadas por análise de variância (ANOVA), seguido do teste de Tukey ou t student. Para a análise estatística utilizou-se o GraphPad Prism ${ }^{\circledR} 6.0$ Software. Sendo o nível de significância mínimo para rejeição da hipótese nula fixado em 5\% ( $\left.p<0.05\right)$.

\section{Resultados e Discussão}

\subsection{Dosagem de Fenóis totais}

A quantidade de fenóis totais para a amostra em estudo foi calculada em 128,54+14,42 mg EAG/g de extrato.

Os compostos polifenólicos englobam várias classes de metabólitos secundários como os flavonoides, os flavonols, os taninos e as antocianinas, esses compostos possuem a capacidade de desenvolver importantes atividades farmacológicas, tendo como principais exemplos as atividade antioxidante e anti-inflamatória (Siddhuraju \& Becker, 2003).

Os resultados da quantificação de compostos fenólicos totais no extrato etanólico das folhas de Moringa oleifera foi

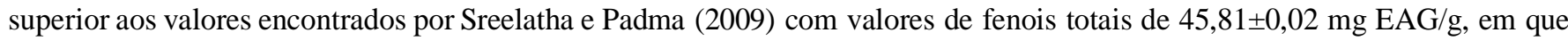
foi utilizado o extrato aquoso das folhas, e com 53,69 \pm 1,00 mg EAG/g mensurados por Nascimentoet al. (2013) utilizando-se o extrato etanólico das folhas.

Os valores encontrados por Gimenis (2015) foram os que mais se aproximaram do teor de fenóis totais encontrados no presente estudo, com 165,58 mg/Eag, no extrato etanólico seco.

O valor encontrado em nosso estudo foi superior ainda aos identificados por Moyo et al. (2012) e Brito et al. (2018), realizados com outra espécie e que atribuíram aos compostos fenólicos atividade antioxidante, anti-inflamatória e gastroprototetora. Entretanto, o teor de compostos fenólicos totais pode variar entre os estudos (até mesmo da mesma espécie), dependendo do metodo extrativo utilizado, estágio de maturação das folhas, solvente utilizado e tempo de extração. Assim, pode ser observável uma moderada variação entre os valores, comparados com outros estudos (Siddhuraju \& Becker, 2003).

\subsection{Dosagem de flavonoides totais}

O conteúdo de flavonoides presentes no EEMo foi de 158,62 mg EQ/100 g de extrato. Valor superior ao encontrado por Teles et al. (2016) que encontrou a quantidade de $85,6 \pm 5,0 \mathrm{mg} \mathrm{EQ} / 100 \mathrm{~g}$ utilizando o extrato hidroalcolico das folhas de M. oleifera. O resultado também mostrou-se com teor superior ao encontrado por Jaiswal et al. (2013), em que foi determinado o valor de 40,5 mg EQ, utilizando o extrato aquoso das folhas jovens. 
A variação apresentada entre valores pode ocorrer por conta de fatores estressantes que induzem ou reduzem a produção de metabólitos secundários pela espécie vegetal estudada, assim, alterações no regime de chuvas, modificações nos componentes nutricionais presentes no solo e o horário de coleta da amostra vegetal influenciam diretamente na quantidade de flavonoides presentes no extrato. Além disso, as metodologias utilizadas para realizar a extração desses componentes, tipo de solvente utilizado e o tempo de extração também podem influenciar nos resultados apresentados (Siddhuraju \& Becker, 2003; Moyo et al., 2012; Sreelatha \& Padma, 2009).

Os flavonoides são compostos polifenólicos sintetizados nas espécies vegetais a partir da via dos fenilpropanoides e apresentam-se com grande diversidade de compostos entre a maioria de especies vegetais superiores, dentre suas atividades está a proteção dos vegetais contra os raios UV, atuam como agente alelopático, possuem atividade antiviral, antioxidante, antitumoral e anti-inflamatória (Simões et al., 1999). O alto valor de teor de flavonoides presentes no extrato das folhas de $M$. oleifera contribui para atribuiçãoda atividade antioxidante pesquisada nos modelos de DPPH.

\subsection{Avaliação da atividade antioxidante}

As diferentes concentrações das amostras do EEMo exibiram atividade antioxidante, sendo o consumo de DPPH diretamente proporcional à concentração do extrato, tendo como o maior potencial de sequestro de radicais livres atribuido à amostra na concentração de $1000 \mathrm{mg} / \mathrm{mL}$ seguido da amostra de $500 \mathrm{mg} / \mathrm{mL}$ com 71,39 e 52,21\% de inibição respectivamente Tabela 1.

Tabela 1 - Avaliação da atividade antioxidante de EEMo.

\begin{tabular}{ll}
\hline EEMo $\mathbf{~ g / m L}$ & \% de inibição \\
\hline 1000 & $71,39 \pm 0,52$ \\
500 & $52,21 \pm 0,19$ \\
250 & $48,63 \pm 0,14$ \\
125 & $48,01 \pm 0,54$ \\
\hline
\end{tabular}

*Os valores apresentam a média \pm E.P.M ( $\mathrm{n}=3$ /grupo). As diferenças entre os grupos foram determinadas por análise de variância (ANOVA), seguido pelo teste de T student.

Fonte: Autores, (2019).

Os valores estão expressos em porcentagem de inibição da oxidação, dessa forma, a atividade antioxiante é avaliada a partir da quantidade DPPH consumida pelo extrato. Assim, quanto maior o sequestro de radicais do DPPH realizada pela amostra, maior seu potencial antioxidante (Alves et al., 2007).

A atividade antioxidante observada pode ser justificada pela alta quantidade de compostos fenólicos e flavonoides presentes no extrato etanólico, tendo em vista que essas substâncias possuem um alto potencial de redução de radicais livres. Essa funçãoé atribuida a estrutura quimica dessas classses de metabólitos secundários onde há grande quantidade de grupos hidroxila conjugados com aneis aromáticos, responsáveis por reações que tornam estáveis os radicais livres presentes no meio, por conta da ressonância presente nos aneis aromáticos. (Oyedemi, Bradley \& Afolayan, 2010).

\subsection{Avaliação da atividade gastroprotetora}

Nesse estudo o grupo controle lesionado apresentou área de lesão ulcerativa de $427 \mathrm{~m}^{2}$. O grupo do lansoprazol e o grupo do EEMo (200 mg/kg) apresentaram proteção gástrica de 66,04 e 48,48 \% respectivamente quando comparados ao grupo controle lesionado (Figura 1). 
Figura 1 - Atividade gastroprotetora do extrato etanólicos das folhas de Moringa oleífera.

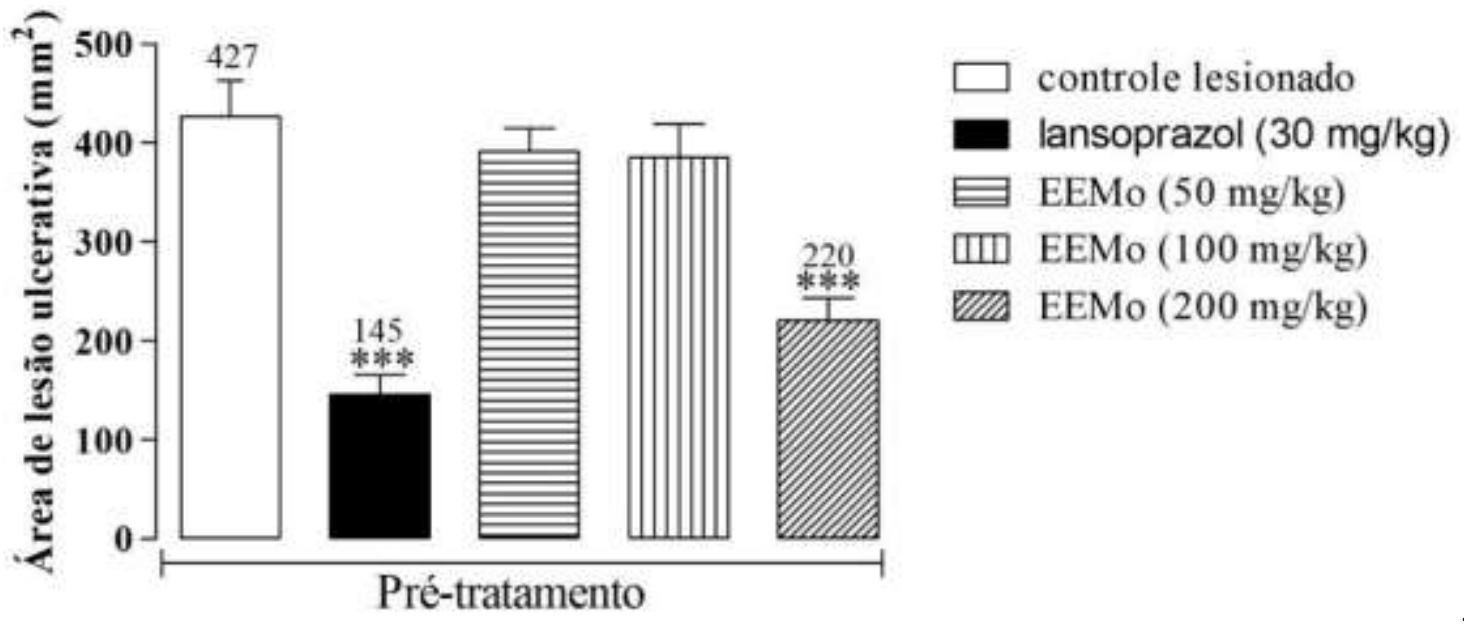

Fonte: Autores.

Os resultados estão organizados em relação à area total ulcerada, sendo comparados o grupo controle lesionado com administração de etanol P.A, o grupo tratado com o fármaco gastroprotetor lansoprazol e os grupos nos quais foram administrados o extrato nas doses de $50 \mathrm{mg} / \mathrm{kg}, 100 \mathrm{mg} / \mathrm{kg}$ e $200 \mathrm{mg} / \mathrm{kg}$.

Considerando o alto teor de compostos fenólicos e flavonoides mensurados no extrato utilizado e a atividade antioxidante apresentada pela amostra, torna-se possível correlacionar a presença desses compostos e a atividade antioxidante com a gastroproteção demonstrada pelo extrato na dose de $200 \mathrm{mg} / \mathrm{kg}$. Tendo em vista que, essas classes de metabólicos secundários são caracterizadas por possuir diversas atividades farmacológicas e, dentre elas, as atividades anti-inflamatória, antioxidante e antiulcerogênica (Simões et al., 1999; Moyo et al., 2012; Zayachikiwaka et al., 2005). Porém experimentos que comprovem seu mecanismo de ação precisam ser realizados.

A capacidade de indução de úlcera gástrica pelo etanol é explicada pelos efeitos agressores provocados na mucosa gástrica, com o aumento de especies reativas de oxigênio e do processo inflamatório (Araújo, 2008). Dessa forma, a presença de compostos que atuam reduzindo essas ações agressoras, sustenta a atividade gastroprotetora observada no presente estudo.

$\mathrm{Na}$ avaliação da atividade gastroprotetora das folhas de Moringa oleifera, realizado por Ijioma et al. (2018) em modelo de úlcera gástrica induzido por aspirina observou-se que o extrato etanólico na dose de $200 \mathrm{mg} / \mathrm{kg}$ não apresentou efeito gastroprotetor relevante, enquanto que o extrato administrado na dose de $800 \mathrm{mg} / \mathrm{kg}$ apresentou efeito gastroprotetor considerável nos animais tratados. Por outro lado, na avaliação de atividade gastroprotetora utilizando o extrato aquoso das folhas, também em modelo de úlcera gástrica induzida por aspirina executado por Mabrok e Mohamed (2019) foi observado atividade gastroprotetora nos animais tratados com o extrato na dose de $300 \mathrm{mg} / \mathrm{kg}$.

O modelo escolhido para a indução de úlcera gástrica foi o uso de etanol P.A., como agente indutor de lesões. Esse é o modelo de primeira escolha em comparação ao modelo de indução por anti-inflamatório, pois o etanol possui a capacidade de alterar uma elevada quantidade de mecanismos fisiológicos gastroprotetores, induzindo assim a formação de úlcera (Possenti et al., 2011). Tem-se então a ação do etanol no muco gástrico, tornando o estômogo mais susceptível ao efeito agressor do ácido cloridrico. Além disso, a administração oral do etanol causa a diminuição dos níveis de prostaglandina e glutationa endogênas, também induzem o aumento da secreção de histamina, geração de estresse oxidativo, produção de leucotrienos e redução de compostos sulfidrilas nas células da mucosa (Ochi et al., 2005; Pacheco et al., 2006; Barros et al., 2008).

O presente estudo apresenta-se como o primeiro a avaliar a atividade gastroprotetora do extrato etanólico da folhas de Moringa oleifera, cultivada no Nordeste do Brasil, por meio do modelo de úlcera gástrica induzida por etanol P.A. 


\subsection{Avaliação de toxicidade}

$\mathrm{Na}$ análise do consumo de água, ração e na mensuração da massa corporea dos animais durante 14 dias, não foram observadas diferenças entre os ratos do grupo controle e o grupo em qual foi administrado a dose de $1000 \mathrm{mg} / \mathrm{kg}$ de massa corporea do extrato. A massa dos órgãos dos animais tratados com $\mathrm{NaCl} 0,9 \%$ e dos animais que receberam a dose de $1000 \mathrm{mg} / \mathrm{kg}$ do extratato etanólico de Moringa oleifera (EEMo) também não diferiram e está representada na Tabela 2.

Tabela 2 - Análise da massa dos órgãos.

\begin{tabular}{lll}
\hline Órgãos $(\mathbf{g})$ & Controle $(\mathbf{N a C l ~ 0 , 9 \% )}$ & EEMo $(\mathbf{1 . 0 0 0} \mathbf{~ m g} / \mathbf{k g})$ \\
\hline Fígado & $9,11 \pm 0,22$ & $9,83 \pm 0,81$ \\
Baço & $0,51 \pm 0,72$ & $0,58 \pm 0,52$ \\
Rins & $1,38 \pm 0,21$ & $1,27 \pm 0,06$
\end{tabular}

*Os valores apresentam a média \pm E.P.M $(n=3 /$ grupo $)$. As diferenças entre os grupos foram determinadas por análise de variância (ANOVA), seguido pelo teste de $\mathrm{T}$ studente comparados ao grupo controle ( $\mathrm{NaCl} 0,9 \%)$.

Fonte: Autores.

A avaliação de alterações sob o sistema nervoso central e autonomo revelaram a ausência de alterações comportamentais nos animais observados.

Nos estudos de toxicidade aguda e sub-aguda realizados por Asiedu-Gyekye et al. (2014), utilizando o extrato etanólico das folhas de Moringa oleifera nas doses de $1000 \mathrm{mg} / \mathrm{kg}$ e $5000 \mathrm{mg} / \mathrm{kg}$ foi relatado que os animais do grupo que recebeu as doses do extrato, apresentaram mais exitação e salivação em relação ao grupo controle negativo, porém com nenhuma alteração histológica apresentada.

\section{Conclusão}

Moringa oleifera apresenta-se como uma espécie vegetal amplamente utilizada empiricamente e uma de suas aplicações na medicina popular está no tratamento de úlcera péptica. Dessa forma, o presente estudo buscou investigar a ação gastroprotetora das folhas dessa espécie para comprovar a efetividade de sua atividade antioxidante e gastroprotetora.

Por meio dos experimentos realizados, tornou-se possível identificar a elevada atividade antioxidante e o alto teor de fenóis totais e flavonoides presentes no extrato das folhas, justificando a atividade gastroprotetora observada no modelo de úlcera gástrica induzida por etanol P.A, comprovando os benefícios da utilização etnofarmacológica com essa finalidade.

Além disso os estudos de avaliação de toxicidade aguda demonstraram que o extrato etanólico das folhas de Moringa oleifera não apresentou alterações comportamentais e fisiológicas que indicassem a presença de toxicidade relevante na dose de $1000 \mathrm{mg} / \mathrm{kg}$. Demostrando o potencial farmacológico presente nessa espécie, sendo então um importante alvo de estudo para o desenvolvimento de medicamentos fitorápicos.

Entretanto, para assegurar a atividade gastroprotetora e a segurança de Moringa oleifera na sua utilização como medicamento fitoterápico, faz-se necessário a execução de mais estudos de atividade antiulcerogênica utilizando outros modelos de indução de úlcera gástrica, com descrição do possível mecanismo de ação gastroprotetor, utilizando diferentes sistemas de solventes para a extração, assim como realizar novos modelos de estudos de toxicidade.

\section{Referências}

Alves, C. Q., Brandão, H. N., David, J. M., David, J. P., \& Lima, L. D. S. (2007). Avaliação da atividade antioxidante de fla vonóides. Diálogos \& Ciência Revista da rede ensino FTC, 12, 1-8. 
Araújo, M. D. S. (2010). Manejo de espécies florestais para produção de madeira, forragem e restauração de áreas degradadas. Caicó: Emparn, 5, 60.

Asiedu-Gyekye, I. J., Frimpong-Manso, S. A. M. U. E. L., Awortwe, C., Antwi, D. A., \& Nyarko, A. K. (2014). Micro and macroelemental composition and safety evaluation of the nutraceutical Moringa oleifera leaves. Journal of Toxicology, 2014, 1-13.

Barros, M. P., Lemos, M., Maistro, E. L., Leite, M. F., Sousa, J. P. B., Bastos, J. K., \& de Andrade, S. F. (2008). Evaluation of antiulcer activity of the main phenolic acids found in Brazilian Green Propolis. Journal of ethnopharmacology, 120(3), 372-377.

Brand-Williams, W., Cuvelier, M. E., \& Berset, C. L. W. T. (1995). Use of a free radical method to evaluate antioxidant activity. Lebensmittel Wissenschaften und Technologie London, 28(1), 25-30.

Brito, S. A., Barbosa, I. S., de Almeida, C. L., de Medeiros, J. W., Silva Neto, J. C., Rolim, L. A., \& Wanderley, A. G. (2018). Evaluation of gastroprotective and ulcer healing activities of yellow mombin juice from Spondias mombin L. PloS one, 13(11), 1-16.

Carvalho, A. S. (2000). Úlcera péptica. Jornal de pediatria, 76(2), 127-134.

Dahot, M. U. (1988). Vitamin contents of the flowers and seeds of Moringa oleifera L. Journal of Biochemistry, 21(1-2), 21-24.

Faizi, S., Siddiqui, B. S., Saleem, R., Aftab, K., Shaheen, F., \& Gilani, A. U. H. (1998). Hypotensive constituents from the pods of Moringa oleifera. Planta medica, 64(3), 225-228.

Gimenis, J. M. (2015). Avaliação da atividade antioxidante, fotoprotetora e antiglicante dos extratos das folhas e flores de Moringa oleífera. Dissertação de Mestrado - Faculdade de Ciências e Letras de Assis - Universidade Estadual Paulista.

Ijioma, S. N., Nwaogazi, E. N., Nwankwo, A. A., Oshilonya, H., Ekeleme, C. M., \& Oshilonya, L. U. (2018). Histological exhibition of the gastroprotective effect of Moringa oleifera leaf extract. Comparative clinical pathology, 27(2), 327-332.

Jahn, S. A., Musnad, H. A., \& Burgstaller, H. (1986). The tree that purifies water: cultivating multipurpose Moringaceae in the Sudan. Unasylva, 38(152), 2328 .

Jaiswal, D., Rai, P. K., Mehta, S., Chatterji, S., Shukla, S., Rai, D. K., \& Watal, G. (2013). Role of Moringa oleifera in regulation of diabetes-induced oxidative stress. Asian Pacific journal of tropical medicine, 6(6), 426-432.

Kalyanakrishnan, R., \& Salinas, R. C. (2007). Peptic ulcer disease. American family physician, 76(7), 1005-1012.

Kangwan, N., Park, J. M., Kim, E. H., \& Hahm, K. B. (2014). Quality of healing of gastric ulcers: natural products beyond acid suppression. World Journal Gastrointest Pathophysiol, 15(1), 40-47.

Kasote, D. M., Katyare, S. S., Hegde, M. V., \& Bae, H. (2015). Significance of antioxidant potential of plants and its relevance to therapeutic applications. International journal of biological sciences, 11(8), 982-991.

Leone, A., Spada, A., Battezzati, A., Schiraldi, A., Aristil, J., \& Bertoli, S. (2015). Cultivation, genetic, ethnopharmacology, phytochemistry and pharmacology of Moringa oleifera leaves: An overview. International journal of molecular sciences, 16(6), 12791-12835.

Lorenzi, H., \& Matos, F. J. (2002). Plantas medicinais no Brasil: nativas e exóticas. Nova Odessa: Instituto Plantarum, 346-347.

Mabrok, H. B., \& Mohamed, M. S. (2019). Induction of COX-1, suppression of COX-2 and pro-inflammatory cytokines gene expression by moringa leaves and its aqueous extract in aspirin-induced gastric ulcer rats. Molecular biology reports, 46(4), 4213-4224.

Matos, F. D. A. (1997). Introdução à fitoquímica experimental. Edições UFC.

Matsui, H., Shimokawa, O., Kaneko, T., Nagano, Y., Rai, K., \& Hyodo, I. (2011). The pathophysiology of non-steroidal anti-inflammatory drug (NSAID)induced mucosal injuries in stomach and small intestine. Journal of clinical biochemistry \& nutrition, 48(2), 107-111.

de Mendonça, M. A., Ribeiro, A. R., Lima, A. K. D., Bezerra, G. B., Pinheiro, M. S., de Albuquerque-Júnior, R. L., \& Cardoso, J. C. (2020). Red propolis and its dyslipidemic regulator formononetin: evaluation of antioxidant activity and gastroprotective effects in rat model of gastric ulcer. Nutrients, 12(10), 2951.

Morton, J. F. (1998). The horseradish tree, Moringa pterygosperma (Moringaceae). A boon to arid lands?. Economic botany, 45(3), 318-333.

Moyo, B., Oyedemi, S., Masika, P. J., \& Muchenje, V. (2012). Polyphenolic content and antioxidant properties of Moringa oleifera leaf extracts and enzymatic activity of liver from goats supplemented with Moringa oleifera leaves/sunflower seed cake. Meat science, 91(4), 441-447.

Mughal, M. H., Ali, G., Srivastava, P. S., \& Iqbal, M. (1999). Improvement of drumstick (Moringa pterygosperma Gaertn.) - a unique source of food and medicine through tissue culture. Hamdard Med, 42(1), 37-42.

Nascimento, J. A., Araújo, K. L. G. V., Epaminondas, P. S., Souza, A. S., Magnani, M., Souza, A. L., Soledade, L. E. B., Queiroz, N., \& Souza, A. G. (2013). Ethanolic extracs of Moringa oleiffera Lam: evaluation of its potencial as an antioxidante additive for fish oil. Journal of Thermal Analysis and Calorimetry, 114(2), 51-56.

Ochi, T., Shibata, H., Higuti, T., Kodama, K. H., Kusumi, T., \& Takaishi, Y. (2005). Anti-Helicobacter pylori Compounds from Santalum álbum. Journal of Natural Products, 68(6), 819-824.

Oecd, T. N. (2002). 420: Acute Oral Toxicity - Fixed Dose Procedure, OECD Guidelines for the Testing of Chemicals, Section 4. OECD Publishing.

Oyedemi, S. O., Bradley, G., \& Afolayan, A. J. (2010). In-vitro and-vivo antioxidant activities of aqueous extract of Strychnos henningsii Gilg. African Journal of pharmacy and pharmacology, 4(2), 70-78. 
Research, Society and Development, v. 10, n. 4, e55210414410, 2021

(CC BY 4.0) | ISSN 2525-3409 | DOI: http://dx.doi.org/10.33448/rsd-v10i4.14410

Pacheco, M. T. B., Bighetti, E., Antônio, M., Carvalho, J. E. D., Rosaneli, C. F., \& Sgarbieri, V. C. (2006). Efeito de um hidrolisado de proteínas de soro de leite e de seus peptídeos na proteção de lesões ulcerativas da mucosa gástrica de ratos. Revista de Nutrição, 19(1), 47-55.

Pal, S. K., Mukherjee, P. K., \& Saha, B. P. (1995). Studies on the antiulcer activity of Moringa oleifera leaf extract on gastric ulcer models in rats. Phytotherapy research, 9(6), 463-465.

Paliwal, R., Sharma, V., \& Pracheta, J. (2011). A review on horse radish tree (Moringa oleifera): A multipurpose tree with high economic and commercial importance. Asian Journal of Biotechnology, 3(4), 317-328.

Popoola, J. O., \& Obembe, O. O. (2013). Local knowledge, use pattern and geographical distribution of Moringa oleifera Lam. (Moringaceae) in Nigeria. Journal of Ethnopharmacology, 150(2), 682-691.

Possenti, A., Carvalho, J. E. D., Monteiro, K. M., Dias, I. M. G., Gibrim, N. F., \& Jacobucci, H. B. (2011). Atividade antiulcerogênica e mecanismo de ação de alimento Fermentado à base de trigo e soja utilizado como alimento Funcional. GED gastroenterol. endosc. dig, 30(4), 125-131.

Sharifi-Rad, M., Fokou, P. V. T., Sharopov, F., Martorell, M., Ademiluyi, A. O., Rajkovic, J., \& Sharifi-Rad, J. (2018). Antiulcer agents: From plant extracts to phytochemicals in healing promotion. Molecules, 23(7), 1751.

Siddhuraju, P., \& Becker, K. (2003). Antioxidant properties of various solvent extracts of total phenolic constituents from three different agroclimatic origins of drumstick tree (Moringa oleifera Lam.) leaves. Journal of agricultural and food chemistry, 51(8), 2144-2155.

Simões, C. M. O., Schenkel, E. P., Gosmann, G., Mello, J. C. P., Mentz, L. A., \& Petrovick. P. R. (2010). Farmacognosia: da planta ao medicamento. 6.ed. Porto Alegre: Editora da UFRGS, Florianópolis: Editora da UFSC.

Somali, M. A., Bajneid, M. A., \& Al-Fhaimani, S. S. (1984). Chemical composition and characteristics of Moringa peregrina seeds and seeds oil. Journal of the American Oil Chemists' Society, 61(1), 85-86.

Sreelatha, S., \& Padma, P. R. (2009). Antioxidant activity and total phenolic content of Moringa oleifera leaves in two stages of maturity. Plant foods for human nutrition, 64(4), 303-311.

Teles, A. R. S., Barreto, L. C. O., Moreira, J. J. S., \& Santos, J. A. B. (2016). Potencial antioxidante do extrato etanólico de folhas da Moringa oleifera lam. XXV Congresso Brasileiro de Ciência e Tecnologia de Alimentos. Gramado - RS.

Woisky, R. G., \& Salatino, A. (1988). Analysis os propolis: some parametersond prodecore for chemical fuality control. Journal. Apic. Res. 37(2), 99-105.

Zayachkivska, O. S., Konturek, S. I., Drozdowicz, D., Konturek, P. C., Brzozowski, I., \& Ghegotsky, M. R. (2005). Gastroprotective effects of flavonoids in plant extracts. J Physiol Pharmacol, 56(1), 219-231. 